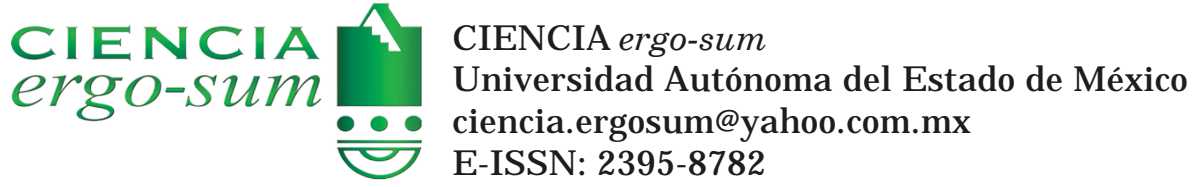

\title{
Semblantes teóricos de Neuroartes: una vía hacia la salud sociovital
}

\author{
García Piedras, Myriam
}

Semblantes teóricos de Neuroartes: una vía hacia la salud sociovital

CIENCIA ergo-sum, vol. 27, núm. 1, marzo-junio 2020|e78

Universidad Autónoma del Estado de México, México

Esta obra está bajo una Licencia Creative Commons Atribución-NoComercial-SinDerivar 4.0 Internacional .

García Piedras, M. (2020). Semblantes teóricos de Neuroartes: una vía hacia la salud sociovital. CIENCIA ergo-sum, 27(1). https://doi.org/10.30878/ces.v27n1a10 


\section{Semblantes teóricos de Neuroartes: una vía hacia la salud sociovital}

Theoretical faces of Neuroartes: A path to socio-vital health

Myriam García Piedras

Instituto Politécnico Nacional, México $\quad$ Recepción: 15 de marzo de 2019

miriam.garcia.piedras@gmail.com

Aprobación: 13 de junio de 2019

\section{RESUMEN}

Se exponen algunos de los planteamientos teóricos de Neuroartes, instituto que tiene como propósito el desarrollo del bienestar socioeconómico, político, cultural y natural-ecológico. Se posicionan algunas interpretaciones de los presupuestos de Everett III y Mensky sobre la mecánica cuántica, elevándose como el paradigma teórico, ya que parte de las relaciones entre la mente, los procesos bioquímicos del sistema nervioso y la conciencia (procesos neuroepistémicos). En este contexto, el arte y la salud se correlacionan a la manera de un binomio, y el arte conduce al despertar neuronal y expansión de los correlatos de la conciencia fortificando la salud individual y sociovital al generar las condiciones en la construcción y transformación de mundos más justos, inclusivos y solidarios.

PALABRas ClaVE: Neuroartes, conciencia, mecánica cuántica, salud, arte.

\section{Abstract}

This article exposes some of the theoretical approaches of Neuroartes. Institute whose purpose is the development of socio-economic, political, cultural and natural-ecological wellbeing. Some quantum mechanics interpretations (Everett and Mensky) are positioned as the theoretical paradigm, since part of the relationships between the mind, the biochemical processes of the nervous system and consciousness (neuroepistemologíc processes). As art and health are correlated in the manner of a binomial, the first leads to the neuronal awakening and expansion of consciousness fortifying individual and sociovital health, generating conditions in the construction and transformation of fairer, more inclusive worlds and solidarity.

KEYWORDS: Neuroarts, awarness, quantum mechanics, health, art.

\section{Presupuestos teóricos de Neuroartes}

El filósofo belga Luc Delannoy fundó el Instituto de Neuroartes, el cual ha desarrollado una propuesta sobre la generación y propulsión del conocimiento que abarca la percepción, la imaginación, la empatía, la creatividad, el razonamiento; todos estos son procesos neuroepistémicos o universales neurocognitivos propios de la morfología cerebral. En otras palabras, las relaciones y la evolución de las facultades del cerebro tienen correspondencia con la generación y expansión de la conciencia,[1] por lo que se elevan como fuentes generadoras en la construcción de otros mundos, idealmente mejores, trascendiendo la idea de un mundo fijo y preestablecido (Delannoy, 2015) y deconstruyendo la concepción de que el mundo es así y no puede ser de otra forma.

Apostar en la posibilidad de construir mundos, idealmente mejores, encuentra una viabilidad teórica en la lectura que realiza Delannoy sobre la mecánica cuántica posmaterialista, principalmente de los físicos Hugh Everett III y de Michael Mensky, así como de algunos presupuestos de la escuela budista Yogacara,[2] trascendiendo el paradigma de la física clásica, la cual concibe el mundo bajo una concepción mecanicista, predeterminada, preexistente y fija (Delannoy, 2017; Pigem 2009). Por el contrario, la mecánica cuántica conduce a la idea de cocreación y apuntala que el sujeto tiene la capacidad de transformar la vida en todas sus dimensiones: políticas, económicas, sociales, ecológicas, artísticas, etc. Neuroartes (2020), a la manera de un manifiesto, apunta: 
1.- El llamado mundo no es una singularidad objetiva pre-existente pública compartida. No tiene fronteras pre-fijadas ni predeterminadas. 2.- El llamado mundo existe como flujo continuo de energías dinámicas no-estructurado (de viscosidades como las califica Edmond Wright) y no como una serie de entidades singulares pre-objetivas. Hablamos de existencia, no de objetos en un mundo pre-existente. Para más claridad usaremos el término inglés nothing- no-thing. Nothing no significa no-existencia; desde este nivel de nada/existencia surgen, percibidos/vividos en cosas, objetos y el mundo tal como lo percibimos. Según Müller la materia sólo puede ser percibida por la mente. Considerar la materia sin que esté involucrada la mente es una creencia dogmática, una idea, un punto de vista filosófico incompleto. La cuestión es entender cómo relacionar la mente con la materia o sencillamente si los debemos relacionar. Nuestra propuesta es epistemológicamente no-objetivista. 3.- No existe realidad si no es vivida a través de la experiencia presente. No podemos separar la realidad como fenómeno físico del sujeto. No podemos iniciar nada si no es a partir de nuestra propia experiencia. La experiencia ocurre en un contexto biológico, actualiza los contenidos de la percepción. La separación sujeto-objeto no es primaria sino secundaria y pragmática. Es una ficción que tiene un fin práctico. El mundo es el resultado no la causa de nuestra percepción. Los mundos que construimos son exteriores a nuestros cerebros mientras las causas neuronales y las sensaciones neuronales son en nuestros cerebros. Hacemos de nuestros mundos fenomenales nuestros mundos reales/físicos. 'El cerebro proyecta sobre el mundo sus percepciones internas, construye su percepción en función de las acciones que prepara' (Berthoz). El monismo reflexivo de Max Velmans habla de realidades fenomenales proyectadas por redes neuronales intracraneales. $\mathrm{Si}$ entretenemos prototipos perceptivos que proyectamos, corremos el riesgo de encerrar individuos y objetos en esquemas a priori, pre-establecidos. La propuesta epistemológica de Neuroartes evita este riesgo. La experiencia perceptiva es una experiencia cerebral y corporal dinámica (Delannoy, 2010: 51-53). 4.- Ante todo, el arte se presenta como una forma de enseñanza: nos permite aprender sobre nuestra propia vida, sobre nuestros procesos perceptivos. El arte habla de nuestra humanidad, de nuestras posibles relaciones con varios mundos, con múltiples realidades; es fundamentalmente una creación intersubjetiva, abre un espacio de diálogo, de creación poética, de esperanza común en el cual invita al público a expresarse. Nos permite imaginarnos e imaginar al otro. El arte nos libera de la presión de pretender compartir una realidad objetiva preexistente. No se trata de aprehender la realidad. Fomentar la práctica artística es fomentar la apertura para construir posibilidades, posibilidades que podemos construir y disfrutar juntos. El arte empodera y genera experiencias vivenciales que muestran que el ser humano sí puede cambiar y cambiar el mundo que estructura y construye. Los artistas tienen entonces una responsabilidad social fundamental. Las obras de arte generan haces de resonancias. 5.- La ciencia y la neurociencia se ocupan de la conciencia discriminatoria y de la co-emergencia. La filosofía se ocupa de los dos niveles de conciencia. Sin la filosofía la ciencia es incompleta. El diseño de nuestras propuestas de intervenciones sociales se basan en la unidad de investigaciones transdisciplinarias: filosofía, neurociencia, biología, psicología, educación, artes, economía. 6.- El paradigma clásico nutre un capitalismo voraz que cosifica los aspectos subjetivos los más profundos del ser humano. Creemos en la necesidad de fortalecer instituciones y comunidades democráticas y humanistas para proponer un nuevo contrato social que reconoce la importancia de un equilibrio entre Ser y Hacer.

La cocreación invita a potencializar la construcción y generación de mundos distintos y alternos. Para ello, es importante potencializar el desarrollo de los procesos neuroepistémicos. Se apuesta por la siguiente idealidad: a mayor ejercitación de cada área cerebral mayor ampliación de las redes neuronales y generación del conocimiento, el cual se entiende como la expansión de los correlatos de la conciencia. En otras palabras, dependiendo de cómo cada sujeto ejercite sus universales neurocognitivos, desarrollará, transformará o ampliará su arquitectura cerebral y por ende expandirá el posible ejercicio de su conciencia incidiendo tanto en su salud corporal como en la mental:

El despertar neuronal y nervioso, basado en la captación recepción, la vivencia interna y la integración de estímulos exteriores por medio de la cognición, una cierta lucidez en la concientización de los procesos neuronales, extraneuronales y nerviosos y de sus resultados, una lucidez que nos lleva a una comprensión progresiva de los mundos construidos y de nosotros mismos (Delannoy, 2015: 32).

Neuroartes estudia la relación entre la mente, la conciencia, la corporalidad y la vida. Niega cualquier escisión o fractura de orden epistémico entre la conciencia-mente y la corporalidad. No hay separación del sujeto con la vida ni mucho menos diferencia entre lo tangible de lo intangible; encuentra en el monismo no-dual de la escuela budista Yogacara y en el monismo reflexivo de Welmans algunos de sus fundamentos teóricos.

La escuela budista Yogacara plantea que todo es mente. Si todo es mente, se puede hacer la siguiente interpretación: lo que aparece se manifiesta en múltiples formas; paradójicamente lo tangible y lo intangible son parte del flujo contínuum de la vida anulando así cualquier forma de epistemología dualista. 
Por su parte, el monismo reflexivo de Velmans (2008) plantea que la supuesta fractura metafísica ha exacerbado el dualismo epistémico conduciendo a la incapacidad de generar un conocimiento integral u holístico de la vida. Para él, no hay separación entre la materia de la mente (tangible-intangible), ya que las relaciones-duales son aparentes. Para él, todo es mente (monismo reflexivo); lo que existe es la manifestación de la mente universal. En la escuela budista Yogacara, como en el monismo reflexivo, la percepción juega un papel fundamental como el proceso neuroepistémico de percibir el flujo de la vida. No es gratuito que Neuroartes encuentra en la percepción el horizonte neuroepistémico, ya que difumina la idea del mundo estático, objetivo y preexistente

Por otra parte, Delannoy considera algunas interpretaciones de la mecánica cuántica, las cuales sostienen que cuando algo se transforma no existe la certeza y la exactitud de en qué se convertiran. Apunta que el observador participa de lo observado, transformándose ambos, y paradójicamente todo tiende a estar unificado diluyendo las diferencias dualistas entre lo tangible de lo intangible, la materia de lo inmaterial, lo etéreo de lo sólido, la naturaleza de la cultura, etc., así que las manifestaciones de la vida se correlacionan entre sí aunque aparenten ser distintas, y confluye en un devenir dinámico que se transforma continuamente: la vida no es estática, objetiva ni preexistente.

\section{Meditaciones sobre la conciencia}

Cajal (1952) estudió la anatomía, el comportamiento de las neuronas y el tejido nervioso; con esto encontró que las neuronas son células conformadas por el cuerpo celular (soma), los axones y las dendritas que cuentan con extensiones causantes de las sinapsis, las cuales producen corrientes eléctricas mediante la concentración de iones de potasio, calcio, cloro, sodio. Aunado a lo anterior, generan información que fluye desde las ramificaciones dendríticas hacia el cuerpo de la neurona, procesan la información para luego dirigirse hacia otros axones produciendo más sinapsis en otras células. En un aproximado, una neurona se puede conectar con otras cincuenta mil. Paradójicamente, las conexiones sinápticas no tienen una ruta determinada. William James nombra a esto plasticidad neuronal. Delannoy (2017), siguiendo a William James, se pregunta ¿por qué las rutas neuronales toman un rumbo y no otro? y ¿cómo se correlacionan las sinapsis con la adquisición y la generación del conocimiento?

Tratando de aproximarse a dichas preguntas, se apuntala que, a pesar de que los neurocientíficos han estudiado las funciones del cerebro, todavía no se tiene un conocimiento absoluto sobre el enrutamiento neuronal. No es gratuito que Roger Bartra (2007) señale que el cerebro humano es un órgano incomprensible para el conocimiento científico, todavía en el tercer milenio. Más aún, no existe un saber que señale los mecanismos neuronales que sustentan o generen las formas del pensamiento ni de la conciencia. Si bien señala gran parte de las funciones cerebrales ocurren en la corteza cerebral, la cual está conformada por una red que conecta a "miles de millones" de neuronas, no se logra explicar los mecanismos neuronales entre el pensamiento y la conciencia y precisamente el desarrollo de la neurociencia y la neurología tiene la pretensión de encontrar los resortes naturales de la mente, así como el funcionamiento del sistema nervioso central.

Mejía (2012) expone la teoría de Karl Lashley quien señala que el cerebro es equipotente, es decir, que cualquiera de sus partes puede realizar las funciones de las demás, y de éstas no se puede hablar de una localización específica. La propuesta anterior es contraria a la teoría de Wilder Penfield, quien afirma que el cerebro es un entramado de conexiones y de funciones cognoscitivas específicas localizadas en zonas muy concretas del cerebro. Para Mejía la actividad neurocerebral se sitúa entre ambas. La primera conduce a pensar en una actividad holística neurocerebral y también existen áreas del cerebro que realizan funciones cognitivas específicas. Si bien existen áreas muy específicas en la morfología cerebral, éstas no actúan solas. A mayor ejercitación neuronal, las redes neuronales se bifurcan (¿aleatoriamente?) y la plasticidad cerebral se amplía. Mejía (2012: 22-23) escribe: 
Se debe señalar que por término medio una neurona del cerebro humano posee entre 1000 y 10000 sinapsis o puntos de contacto con las neuronas más próximas. Si el cerebro humano tuviera una sola sinapsis -lo que correspondería a un individuo de monumental estupidez- no se podría alcanzar más que dos estados mentales. Pero el caso es que el cerebro humano contiene alrededor de 1013 sinapsis, por lo que el número de estados mentales que puede alcanzar el hombre es de 21013 , es decir, 2 multiplicado por sí mismo diez billones de veces. Se trata de una cifra irrepresentable, mucho mayor por ejemplo, que el número de partículas elementales que existe en todo el universo. Debido a esta ingente cantidad de configuraciones cerebrales funcionalmente distintas no puede haber dos hombres iguales.

Lo más asombroso: cada cartografía cerebral es única, pues va construyendo su propio mapeo cerebral. Cada cerebro tiene sus propias rutas sinápticas, lo cual aún sigue siendo desconocido, tan desconocido el cómo y el por qué una neurona se conecta a otras a pesar de que puedan estar lejos entre sí. Y con esto se explica de alguna forma la teoría del cerebro equipotente de Karl Lashley (cada cerebro es único en su mapeo neuronal).

Neuroartes apuesta a que la plasticidad y el mapeo cerebral pueden ser un potencializador en la construcción de la arquitectura cerebral debido que se ejercita el sistema nervioso y con esto se genera y se adquiere mayor conocimiento. En otras palabras, a mayor ejercitación de las áreas cerebrales mayor plasticidad cerebral y mayor adquisición del conocimiento. Neuroartes encuentra un vaso comunicante con la concepción teoría del cerebro equipotente y con la teoría de la conciencia cuántica.

Para Delannoy (2005), la conciencia cuántica genera una de las posibles respuestas a la actividad cerebral, la cual se manifiesta cuánticamente, esto es, no-determinista y no binaria o computacional desterrando cualquier teoría del reduccionismo mental y computacional. La actividad del pensamiento y de la conciencia no se reducen ni pueden analogar a una computadora ni mucho menos al pensamiento binario. La actividad cerebral es mucho más compleja, por eso se apuesta por la conciencia cuántica.

\section{CONCIENCIA CUÁNTICa: OBJETIVA Y SUbJetiva}

Penrose (2004) es uno de los científicos que ha estudiado a la consciencia cuántica. Se opone a la teoría de la mente computacional bajo el argumento de que los estados de la mente consciente surgen bajo los parámetros de la mecánica-cuántica mediante el colapso de la función de onda generándose la mente-consciente, paradójicamente en el espacio-tiempo cuántico como en el espacio-tiempo clásico, lo cual no puede reducir ni representarse bajo algoritmos computacionales. Por eso está en contra de la mente-computacional. Esta idea que desarrollara posteriormente en la teoría de la reducción objetiva orquestada (Hameroff y Penrose, 2014) postulan que la conciencia es inherente en el devenir del universo-no-computable; existe una correlación (intrínseca) entre la consciencia y el universo. Precisamente, las leyes de la física clásica se correlacionan con el reino de la mecánica cuántica.

Dicha teoría encuentra una liga comunicante con el postulado de Neuroartes en relación con la no-existencia (no-thing) del mundo fijo, preexistente, estático y mecanicista. Sin embargo, Neuroartes se desliga de la teoría de la reducción orquestada, ya que ésta última sigue engarzada en el discurso de la cientificidad objetivista menospreciando el terreno de la subjetividad y por ende la idea de la cocreacion. Esta última destierra la idea de qué el espectador es pasivo; por el contrario, la generación del conocimiento es activo y el sujeto es cocreador y transformador del mundo anulando la idea de que el mundo es así y no puede ser de otra forma, ya que según todo el universo opera por las leyes cuánticas y por eso el conocimiento incide en la experiencia vital del sujeto. No es gratuito que apuesta por el subjetivismo cognoscitivo y los qualia se posicionan como uno de los ejes teóricos en tal propósito.

Neuroartes retoma algunos de los planteamientos de Mensky (2013), quien a su vez retoma al físico Hugh Everett III, según el cual existen otros universos (multiversos) y también paralelos que sólo operan por leyes cuánticas (no mecánicas). En otras palabras, las leyes de la mecánica cuántica operan en el reino de lo micro y de lo macro anulando el colapso de la medición de onda, desterrándose el paradigma de la física clásica; el universo no se concibe como predeterminado y objetivo, por el contrario es no-material o también llamado universo posmaterial. 
Para Hugh Everett III, el mundo cuántico es una colección de partículas que interactúan en más de dos estados o superposiciones, las cuales no son medibles. La idea de los multiuniversos paralelos permite correlacionar la idea de la cocreación exacerbando el papel activo del sujeto. Para Hugh Everett III y Mensky el universo y la conciencia se rigen por las leyes de la mecánica cuántica, y el quantum (valor mínimo de medición a nivel subatómico) genera las condiciones de apertura de la conciencia a la realidad cuántica. Se puede decir que los qualia son la antesala del conocer y la comprensión subjetiva de la vida. Por ello, Neuroartes encuentra en estos la condición necesaria para la vivencia interna del conocimiento irrumpiendo con la idea de que éste requiere fundarse en la objetividad, en lo claro y distinto, alejado de la subjetividad.

Es importante señalar que Neuroartes no desdeña el discurso cientificista de orden objetivo si bien lo encuentra válido, aunque éste no considera la intimidad de la conciencia y, por ende, no toma en consideración que el conocimiento invita a transformar la conciencia del sujeto. Por otra parte se pregunta: jacaso los estados de la conciencia puede ser medibles? Para Neuroartes la vivencia no se puede medir cuantificable ni unívocamente, por eso los qualia se posicionan como uno de los ejes irruptores del conocimiento objetivista, ya que el conocimiento es producto de experiencias subjetivas. La conciencia cuántica se experimenta en la subjetividad no en el conocimiento objetivo. Neuroartes encuentra en Everet III y en Mensky la fundamentación de la conciencia cuántica de orden subjetiva, las bases metodológicas en redimensionar el sentido por la generación y la construcción del conocimiento y con esto se eleva el conocimiento subjetivo como un horizonte vital, el cual propicia y propulsa algunas transformaciones en aras del bien colectivo debido que el mundo interpela con la conciencia y no es ajeno del sujeto.

Delannoy sigue a Mensky: apuesta a que el conocimiento debe ser introspectivo y a la vez apertura a la vida. Los qualia son la relación entre la subjetividad experiencial (de la vivencia) con la realidad. Ningún sujeto percibe, imagina, experimenta, razona, abstrae, empata de igual forma (unívoca) que los otros. Cada sujeto experimenta en la intimidad de su conciencia una forma muy particular de vivir, sin excluir la capacidad en relacionarse con los demás (diría yo: nos relacionamos con los otros de forma análogas, no unívocas ni equívocas). En la medida de que cada sujeto conoce y experimenta a la vida desde la intimidad de su conciencia, su cartografía cerebral y corporal se modifican, expande y hace experiencia tanto de sí mismo como de la vida. Precisamente la educación de orden estética y artística potencializa y es una de las mejores vías de tal propósito.

\section{El ARTE COMO FLUJo PERCEPTIVO}

Delannoy estudia la percepción. Encuentra en los presocráticos, en Protágoras, Heráclito, Sexto Empírico, los estoicos, y en algunos fenomenólogos como Merleau-Ponty, que la consideran como la vía neuroepistémica capaz de conectar la subjetividad de la conciencia con el flujo de la vida y ser el eje rector en el despertar neuronal. Si el sujeto es capaz de captar el flujo de la vida, su plasticidad cerebral se expande ampliando los estados de los correlatos de su conciencia. En otras palabras, la plasticidad cerebral es una vía de autoconocimiento y expansión de la conciencia.

Por otra parte, Bartley (1980) entiende la percepción como una conducta adaptativa mediante la adquisición de los conocimientos del medio por el desarrollo y la ejercitación de las energías físicas que estimulan a los sentidos; Neuroartes también comparte esta idea y la entiende como "la adaptación de las características fisiológicas, afectivas, comportamentales heredadas que se desarrollan de manera confiable en un organismo para aumentar sus chances de sobrevivir y de reproducirse" (Delannoy, 2013: 11). Se puede decir la energía y la percepción se corresponden mediante la captación de las energías externas que estimulan a los sentidos internos generando un mayor número de sinapsis cerebrales. El sujeto percibe vivencialmente su entorno. "No existe la realidad si no es vivida a través de la experiencia. No podemos separar la realidad como fenómeno físico del sujeto. No podemos iniciar nada si no es a partir de nuestra experiencia" (Delannoy, 2015: 144). 
Es importante señalar que la percepción es el puente entre las energías internas (qualia) con las externas, lo cual produce la emisión de noradrenalina, adrenalina y norepinefrina regulando el área subcortical y produciendo emociones en la mayoría de los casos. A su vez, ejercita la glándula del hipotálamo, cuyo centro es la amígdala que activa el sistema nervioso autónomo y las percepciones en el ser humano (Mora y Sanguinetti, 2007). Por su parte, Mejía (2012: 86-87) escribe:

La interpretación de la información física en mensajes informativos que el sistema nervioso puede utilizar es llamada proceso de transducción sensorial. En la vida cotidiana el ser humano debe adaptarse a la variedad de estructuras de energía. Muchas de estas se producen en el medio externo, pero algunos resultan de cambios en los órganos internos. Las primeras son generalmente llamadas estímulos del medio, mientras las últimas son llamadas estímulos de estado (en alusión al estado del organismo).

De tal modo que la percepción comienza a organizarse a nivel de los sentidos (sensación), su organización continúa desarrollándose a medida que los impulsos o patrones de impulsos ascienden al cerebro. Esto da pie a la siguiente etapa perceptual. Cuando los impulsos nerviosos llegan al cerebro, puede ocurrir una de dos cosas: el cerebro puede actuar como un relevo y una estación receptora y transmitir la información al sistema de respuesta, completando así el acto de la percepción; o puede, además seleccionar, reorganizar y modificar la información antes de transmitirla al sistema de respuesta. El cerebro hace las dos cosas, y esto es cada vez más evidente en la medida en que el ser humano asciende en la pirámide del reino animal (progresión filogenética) o en la medida en que se pasa de la etapa de infante a la etapa de adulto experimentado (progresión ontogenética). La última etapa de salida es simplemente prolongación de la tercera etapa. De tal modo que en esta última etapa se puede aseverar que la percepción ha ocurrido, cuando la persona dice verbalmente o a través de cualquier otro índice conductual que ha percibido propiedades del mundo exterior, tales como un color rojo, una línea corta, una cara familiar, una composición musical en acción, la solución de un problema, etc.

Posicionar a la percepción como la energía neuroepistémica que capta el flujo energético y dirige a los qualia al plantear la posibilidad de que ésta accede a los diferentes niveles de la realidad, desde la subjetividad, lo que desdibuja la idea de qué la razón es la facultad por excelencia y que sólo ésta puede representar de forma clara y distinta, esto es, la representación del mundo fijo, preexistente y objetivo. Con ello la percepción se eleva como otra vía del conocimiento posicionando el flujo energético como la parte constitutiva de la vida.

A su vez, en el reino del arte, la percepción juega un factor fundamental en la ejecución como en la contemplación artística. En una obra metafóricamente convergen la visión íntima del artista, del ejecutante, del espectador y de quienes la experimentan compartiendo todos ellos una vivencia de la obra, permitiendo generar una forma de conciencia colectiva y a su vez subjetiva y coligiendo a la obra con todos los actores, a la manera de un comunión colectiva que gesta una expansión del conocimiento, gracias a que la percepción genera un flujo de la realidad en distinto niveles. No es gratuito de que Neuroartes apuesta por el arte y la percepción en la captación de esto niveles y así poder transformar la realidad ( y a su vez, el despertar neuronal se ejercita). Un claro ejemplo es el siguiente:

Ante todo, la traducción del volumen al plano, de la tridimensionalidad a la bidireccionalidad implica una inevitable transmutación de la percepción ingenua al arte pictórico. Si a ello se agrega -como diferencia específica- el papel del tiempo, entonces el cubismo propone una verdadera revolución en el orden de la propuesta de percepción y una nueva teoría concomitante. El lienzo muestra, no el resultado de la percepción ingenua casual, contingente, siempre, "ángulo objetivo de visión”, captación parcial de un objeto. Se trata ahora de una "percepción conceptual” - por así decir-. Se trata de una nueva manera de ver un objeto, en cuanto el cuadro reconstruye simultáneamente todas las superficies, planos, que la percepción común registra sucesivamente (Lapoujade, 2014: 317).

\section{El ARTE-SALUd COMO RESARCIDORES DEL DUALISMO}

Gerald Edelman (1992) es uno de los primeros científicos que cuestiona el dualismo metafísico formulado por Descartes, ya que éste produjo una escisión entre la mente del cuerpo. Tal fragmentación ha exacerbado el dualismo cognoscitivo repercutiendo en la incapacidad de generar un conocimiento integral holístico exacerbando el 
dualismo metafísico. Se cree que el ejercicio de la razón tiene que ver poco con los sustratos biológicos. El reino de la razón (intangible) no encuentra relación con lo tangible (material), produciendo problemáticas complejas. El biólogo chileno Maturana (2009: 59) señala:

En la actualidad vivimos una vida fragmentada que trata a algunas experiencias como la experiencia estética como algo especial y nos cegamos a sus fundamentos biológicos. Sólo los animales como nosotros que existimos en conversaciones son lo que pueden tener lo que he llamado experiencias estéticas, pero los aspectos de la vida que se distinguen en la distinción de la experiencia estética ocurren como parte de la simple vida. Sólo cuando se interfiere con las coherencias de las relaciones de un organismo y su medio, se distorsionan los fundamentos de lo que distinguimos cuando distinguimos una experiencia estética y experimentamos la fealdad. Un desierto es hermoso, pero una tierra que se ha contaminado, deforestado o dañado ecológicamente no es hermosas, es fea, rompe nuestro bienestar mientras nos lleva a un área de nuestro espacio psíquico que destruye la armonía de las coherencias de nuestra vida.

A mayor fragmentación, es poco probable que el sujeto sea consciente de sí como de su entorno. Se apunta que la salud no es exclusiva de la biología, pues existen enfermedades psíquicas y sociales y, se arriesga a decir, hasta espirituales. El dualismo recrudece la enfermedad, por ende la salud se suscribe en el terreno del conocimiento al entenderlo como el desarrollo de todas las facultades neuroepistémicas que inciden en la conciencia y en lo corporal-motriz.

A mayor ejercitación de los procesos neuroepistémicos, mayor plasticidad cerebral, mayor expansión de la conciencia y mayor interacción con el entorno en todos sus ámbitos: personal, socioeconómico, artístico, ecológico, etcétera. No es gratuito que Neuroartes plantea que no existe separación entre el reino de lo bioquímico de lo cultural. El arte y la salud se posicionan como un binomio-correlacional capaz de suturar el dualismo cognoscitivo y metafísico debido que potencializan las capacidades neuroepistémicas incidiendo en el despertar neuronal e idealmente se generan las condiciones de una salud integral.

Los correlatos entre lo bioquímico con la conciencia invita a plantear que "las estructuras biológicas (las categorías del conocimiento de nuestra capacidad de conocer) son innatas. [...] El conocimiento es el resultado de la interacción entre nuestro organismo con el mundo exterior" (Delannoy, 2015: 57), por lo que el supuesto dualismo es aparente. Así, el arte y la salud tienen efectos en el despertar neuronal generando un mayor equilibrio de los procesos homeostáticos (salud) incidiendo (idealmente) en sociedades mas equilibradas en todos los órdenes de la vida. El arte se posiciona como la pedagoga de orden estética-artística capaz de propulsar el despertar neuronal-mental y extraneuronal-mental incidiendo en la expansión de la conciencia y propulsando una conciencia holística que tienda hacia la salud personal, social y ecológica.

\section{Pautas para una salud socio-Vital}

Para el discurso científico dominante, el arte y la salud son saberes con métodos distintos, lo que produce un dualismo metodológico y disciplinar difuminando la posibilidad de vincular ambos saberes y con esto se maximiza la especialización disciplinar.

Gadamer (1996) sostuvo que la medicina moderna no contempla el horizonte holístico de la salud y de la enfermedad. Para el hermeneuta, la salud y la enfermedad tienen correlatos psicológicos y somáticos; sin embargo, el método positivista fragmenta el reino de la bioquímica del reino de la psique, producto de la especialización. Por el contrario, Neuroartes, igual que Gadamer, busca resarcir el dualismo metodológico apostando por un panorama holístico e integral de la salud y de la enfermedad. La salud y la enfermedad se correlacionan con los estados de la conciencia y éstos con el entorno; por eso Neuroartes apuesta por la transdisciplina.

La transdisciplina tiene como propósito la comprensión unitaria del mundo. Siguiendo al rumano Basarab (1996), quien toma el prefijo trans, indica: lo que está a la vez entre las disciplinas, a través de las diferentes disciplinas y más allá de toda disciplina. La transdiciplina es una metodología epistémica que permite vincular a 
las diferentes áreas del conocimiento resarciendo el dualismo disciplinar y metodológico. Si bien Basarab tiene como fundamento teórico a la física cuántica, con esto plantea que existen varios niveles de la realidad, los cuales se conectan por los flujos de la energía.

Ahora bien, si la percepción estructura y capta los flujos de la energía, ayuda a la conducta adaptativa y de sobrevivencia, entonces permite conectar a los diferentes niveles de la Realidad ampliando la capacidad de sobrevivencia mediante la expansión de la conciencia. En otras palabras, la percepción es capaz de potencializar la búsqueda de los patrones, modificaciones, sensaciones y procesos cognitivos. El flujo de la energía percibe a los diferentes niveles de la realidad que paradójicamente puede ser construida o modificada por la cocreación del sujeto. Y con esto Neuroartes posiciona a la percepción como captadora de los flujos o estancamientos de la energía que propulsa a la salud o en su defecto a la enfermedad.

La adaptación, sobrevivencia, supervivencia o, en su defecto, la inadaptación, extinción o muerte son los flujos o estancamientos de la energía tanto personal como colectiva. También el arte juega un papel de sobrevivencia a nivel transhistórico que lleva a Delannoy (2013: 11) a preguntar: “¿Es el arte una adaptación o un derivado de la adaptación? ¿Es posible una explicación darwiniana de las artes? ¿Poseen las artes ventajas para nuestra sobrevivencia y nuestra reproducción?” La percepción, el arte y la salud encuentran así una liga conectiva como fuente de supervivencia tanto personal como colectiva. La salud o la enfermedad simbolizan los flujos o estancamientos de la energía. El arte genera vida con su enorme poder transformativo y creativo, potencializa, ejercita y desarrolla todas las funciones cerebrales:

El arte es un proceso; cuando estamos en presencia de una obra de arte, no se trata de encontrarse en un estado de «felicidad». $\mathrm{Si}$ es lo que buscamos, es mejor tomarse una pastilla. El arte involucra la atención en la búsqueda de patrones. Esta búsqueda conlleva modificaciones de redes cerebrales involucrados en la sensación y la percepción (cognición). El arte es un juego individual y social que genera creatividad sin límite de tiempo. Como juego cognitivo el arte aumenta nuestras habilidades cognitivas, nuestras sensibilidades, nuestra imaginación. Cuando estamos en contacto con obras de arte buscamos siempre los patrones que nos estimulan, mientras más patrones buscamos y encontramos, más desarrollamos habilidades para seleccionar lo que nos conviene como organismos. La manifestación de los patrones de los mundos del arte con los cuales estamos en contacto se observa en nuestros cerebros. El artista crea ante todo desde su propia biología, su mente, sus pensamientos, sus actos (Delannoy, 2013: 13).

Los flujos o estancamientos de energía encuentran un vaso comunicante con la siguiente idealidad: la salud simboliza la perpetuación de la justicia, la equidad, la belleza, solidaridad, etcétera, mientras que la enfermedad reproduce y recrudece la violencia, la injusticia, la explotación social y ecológica. Los índices de salud o de enfermad tanto personal como sociopolítica se elevan como indicadores del amor o desprecio por el arte. En otras palabras, las manifestaciones artísticas fungen como indicadores sociopolíticos que reflejan parte de la época de dónde emergen. Metafóricamente asemejan a un espejo, el cual proyecta la salud o la enfermedad psicosocial.

\section{LOS QUALIA COMO GENERADORES DE LA SALUD SOCIO-VITAL}

Uno de los debates actuales a nivel epistemológico reside en problematizar si la adquisición del conocimiento genera o no una experiencia subjetiva. Si se apunta a que sí, entonces la objetividad del positivismo se pone en tela de juicio. Neuroartes enfatiza la subjetividad de la conciencia (qualia) y apunta a que la adquisición y generación del conocimiento requiere vincular el reino de lo orgánico-biológico con el reino de la conciencia, el mundo de lo objetivo con el mundo de lo subjetivo, confluyendo y transformándose entre sí:

Por muy detallada que sea la descripción de los procesos físicos subyacentes, es difícil concebir de qué manera el mundo de la experiencia subjetiva -ver el color azul, sentir la sensación de calor- puede surgir de eventos puramente físicos. Y, sin embargo, en una era en la que la obtención de imágenes del cerebro, la anestesia general y la neurocirugía son comunes sabemos que el mundo de la experiencia consciente depende fuertemente del delicado funcionamiento del cerebro. Nos percatamos de que la 
conciencia, en todo su esplendor, puede quedar anihilada por una minúscula lesión o un leve desequilibrio químico en ciertas partes del cerebro. De hecho, nuestra vida consciente resulta anihilada cada vez que nuestro cerebro cambia y dormimos sin soñar. Sabemos también que nuestra conciencia propia es, en un sentido profundo, todo lo que hay. La cúpula celeste y los centenares de cosas visibles que se encuentran bajo ella, incluido el propio cerebro -el mundo, en definitiva existen, para cada uno de nosotros, sólo como parte de nuestra conciencia, y desaparecen con ella. El enigma por antonomasia, de qué modo se relaciona la experiencia subjetiva con ciertos eventos descriptibles objetivamente, es lo que Arthur Schopenhauer genialmente denominó el nudo del mundo (Edelman y Tonini, 2002: 6).

El nudo del mundo es una especie de intersticio epistémico-vivencial que recuerda la imposibilidad de ser y de sentir en totalidad. Aunque cada uno esté correlacionado con los otros y no experimenta el ser del otro, paradójicamente cada quien experimenta a la vida de forma íntima aun cuando esté colegido con los demás.

Precisamente los qualia tienen la potencialidad de correlacionar la intimidad de la conciencia con la vida; simbolizan un horizonte de apertura hacia la vida. También se entiende como eventos que acaecen en el tiempo en la subjetividad, en las experiencias íntimas que paradójicamente inciden en la ejercitación neuronal, en la plasticidad y en el mapeo cerebral. La paradoja reside en que son eventos intangibles que se encarnan en el reino de lo orgánicos. En otras palabras, las funciones cerebrales producen estallidos en la subjetividad de la conciencia (qualia); por ejemplo, cuando alguien rememora un acontecimiento puede detonar emociones, afectos, pasiones, etcétera. Algo análogo experimenta el artista en su proceso creativo cuando por ejemplo traza alguna línea o genera (reproduce) sonidos, escribe palabras, las cuales están cargadas de una energía que surge desde la intimidad de su conciencia, de su morfología cerebral manifestándose en la obra de arte: correlación energética entre el mundo interno con el mundo externo. Si bien cada quien experimenta en la intimidad de su conciencia (cerebral-corporal-existencial), los sonidos, colores, las tonalidades, los sabores, las texturas, experiencias también conectan con el ser de la vida generándose una especie de flujo entre la intimidad de la conciencia con el mundo externo; sin los sonidos, los trazos, las palabras, los colores, las texturas sería cuasi impensable que se produjese el proceso creativo. Lo anterior plantea la coexistencia de los distintos planos de la realidad que confluyen entre sí el mundo íntimo del artista, de los intérpretes, de los espectadores, la obra de arte como también el reino de los materiales: el lienzo, los sonidos, las pinturas, etcétera, produciéndose así una correlación activa entre todos a la manera de un diálogo vital. Retomando los qualia, se puede decir que cada uno de los actores, artistas, espectadores e interpretes vivencian su experiencia estético-artística en la intimidad de su conciencia y no externa a ella. Delannoy (2015: 345-346) sigue a Richard Gregory y escribe:

Los qualia musicales son impresionistas. Son impresiones únicas como la experiencia vivida de los gestos de un músico, de la acústica de la sala de conciertos, de la sensación y percepción del brillo del sonido, de la impresión de un timbre, un vibrato, lo chillón de notas altas, el tono de una voz, una armonía particular, el efecto de la mezcla de samplers con sonidos acústicos, y también de lo retardos, de las variaciones, los motivos, los sonidos llamados sucios en las improvisaciones del free jazz, los eventos melódicos y rítmicos. La música genera también qualia extramusicales, ya que nos remiten a elementos de nuestra biografía que nos son propiamente musicales.

\section{REFLEXIONES ¿FINALES?}

Neuroartes concibe el conocimiento como un horizonte experiencial e íntimo, expansivo de la conciencia. Es apertura a la vida. Niega la idea de que el conocimiento requiere ser objetivo, claro y distinto, ya que poco le dice al sujeto sobre sí mismo como de la vida. Neuroartes posiciona a la percepción como el horizonte neuroepistémico capaz de generar la apertura a los distintos niveles de realidad, y entiende a ésta última como la vivencia de cada actor. El conocimiento requiere ser una especie de horizonte experiencial. La experiencia se enarbola como una de las condiciones en la construcción y generación del conocimiento vital y con esto la conciencia se expande, condición para la transformación de la vida en todos sus órdenes: ecológico-natural, sociopolítico y cultural. $\mathrm{El}$ arte se posiciona como una pedagogía práctica a tal propósito y tiene la potencialidad de ejercitar los procesos 
neuroepistémicos expandiendo la intimidad de la conciencia del sujeto en la medida que hace experiencia de la vida por el arte. El arte es apertura a la vida y puede colegir las partes con el todo. Un botón de muestra: una pintura es la visión íntima del artista, es la vivencia del intérprete y de los espectadores, así como también lo son los materiales orgánicos, los sonidos, etcétera.

Neuroartes niega cualquier forma de dualismo. No hay separación (metafísica) entre la naturaleza-cultura o mente-cerebro; por el contrario, se corresponden, lo cual invita a plantear una pedagogía que propulse a la salud como el bienestar personal y colectivo no sólo desde lo biológico sino más bien de forma integral u holística a la manera de una salud biosocio cultural. En otras palabras, lo natural-bioquímico se encarna en lo socio-político-cultural y viceversa. Y con esto se propulsan políticas integrales que inciden en la propulsión del mejoramiento sociopolítico y natural-ecológico.

No es gratuito que Neuroartes apuesta por la construcción de mundos inclusivos, justos, solidarios, equitativos en todas sus dimensiones: económicas, políticas, sociales, ecológicas y existenciales. Precisamente el binomio arte-salud se eleva como una vía capaz de trascender el economicismo-pragmático del capitalismo salvaje, el cual tiende a reducir casi todo a mercancía. Por ello, el arte y la salud se posicionan como un horizonte neuroepistémico y psicopedagógico que invita a la perpetuación de la vida.

\section{ReFERENCIAS}

Bartley, H. (1980). Principios de percepción. México: Trillas.

Bartra, R. (2007). Antropología del cerebro. La consciencia y los sistemas simbólicos. México: FCE.

Battaner, E. (2001). Introducción a la astrofísica. España: Alianza.

Cajal, R. (1952). Neuronismo o reticularismo, las pruebas objetivas de la unidad anatómica de las células nerviosas. España: Instituto Ramón y Cajal.

Basarab, N. (1996). La transdisciplinariedad, Manifiesto. México: Multiversidad Mundo Real Edgar Morin, A.C.

Chatterjee. (1975). The Yogācāra Idealism. Delhi.

Delannoy, L. (2013). La percepción. Notas de Cursos. Neuroartes. Chile

Delannoy, L. (2015). Neuroartes, un laboratorio de ideas. Chile: Metales Pesados.

Delannoy, L. (2017). Una cuestión de conciencia. Chiles: Metales Pesados.

Edelman, G. (1992). Bright Air, brilliant fire: On the matter of the mind. Basic Books.

Edelman, G y Tononi, G. (2002). El universo de la conciencia. Cómo la materia se convierte en imaginación. Crítica.

Gadamer, H. G. (1996). El estado oculto de la salud. Barcelona: Gedisa.

Lapoujade, M. N. (2014). Homo Imaginans I. México: BUAP.

Mejía, T. (2012). Fundamentos psicopedagógicos. México: Red Tercer Milenio.

Maturana, H. (2009). La realidad: ¿objetiva o construida? I Fundamentos biológicos de la realidad. España: UIA-ITESO-Anthropos.

Mensky, M. (2013). Everett interpretation and quantum concept consciousness. NeuroQuantology, 11(1). Disponible en https://www.neuroquantology.com.index.php/journal/article/.../63

Mora, F. y Sanguinetti, A. M. (2007). Diccionario de Neurociencia. España: Alianza

Neuroartes. (2020). Neuroartes. Disponible en https://www.neuroartes.com/ 
Penrose, R. (2004). La mente nueva del emperador. En torno a la cibernética, la mente y las leyes de la física. México: FCE.

Pigem, J. (2009). Buena crisis: hacia un mundo postmaterialista. España: Karirós.

Hameroff, S., \& Penrose, R. (2014). Consciousness in the universe: A review of the 'Orch OR' theory, Physics of Life Reviews. https://doi.org./10.1016/j.plrev.2013.08.002.

Wilber, K. (2016). El cuarto giro: evolucionando hacia un budismo integral. Barcelona: Kairós.Velmans, M. (2008). Reflexive monism. Journal of Consciousness Studies, 15(2), 5-50.

\section{Notas}

[1] En este artículo se entiende la conciencia como "tener conciencia: awareness, diferente de hacer conciencia de algo como consciousness". El verbo hacer conlleva una transformación subjetiva de la consciencia, mientras que tener consciencia plantea un verbo externo a la acción del sujeto.

[2] La escuela Yogacara enfatiza el "todo es Mente” y lo entiende como la sucesión, corrientes o actos del conocimiento, por lo que no existe algo eterno ni permanente, todo fluye a la manera de una corriente perceptual, mientras lo que "aparece" es ilusorio e insustancial, niega cualquier forma del pensamiento dual debido que el sujeto es parte del objeto y lo percibe como parte del flujo de la mente (Chatterjee, 1975; Wilber, 2016).

\section{BY-NC -ND}

